Supporting Information for:

\title{
Optical Ion Sensing Platform Based on Potential-Modulated Release of Enzyme
}

\author{
Jiawang Ding, Enguang Lv, Liyan Zhu, and Wei Qin* \\ Key Laboratory of Coastal Environmental Processes and Ecological Remediation, \\ Yantai Institute of Coastal Zone Research (YIC), Chinese Academy of Sciences \\ (CAS); Shandong Provincial Key Laboratory of Coastal Environmental Processes, \\ YICCAS, Yantai, Shandong 264003, P. R. China. *E-mail: wqin@yic.ac.cn. Fax: \\ +86535 2109000 .
}

\section{Contents}

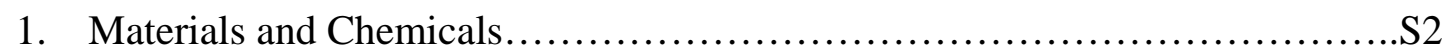

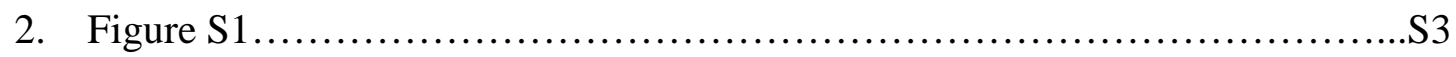

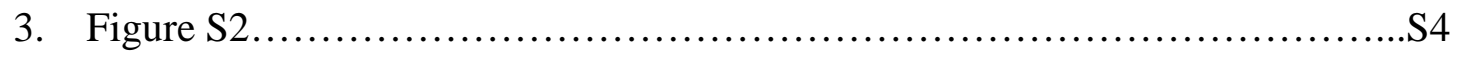

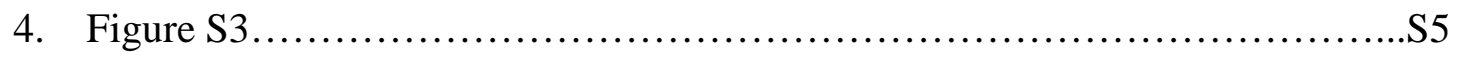

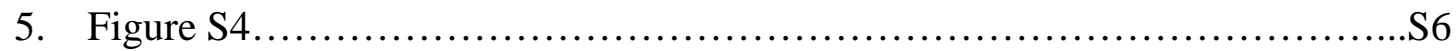




\section{Materials and Chemicals}

Alginic acid sodium salt from brown algae, sodium sulfate (anhydrous, 99\%), ferrous sulfate and hydrogen peroxide were obtained from Sinopharm Group Co., Ltd. Tetrahydrofuran (THF) was freshly distilled prior to use. All other reagents were analytical grade or better. Aqueous solutions were prepared with freshly deionized water (18.2 M $\Omega \mathrm{cm}$ specific resistance) obtained with a Pall Cascada laboratory water system. 


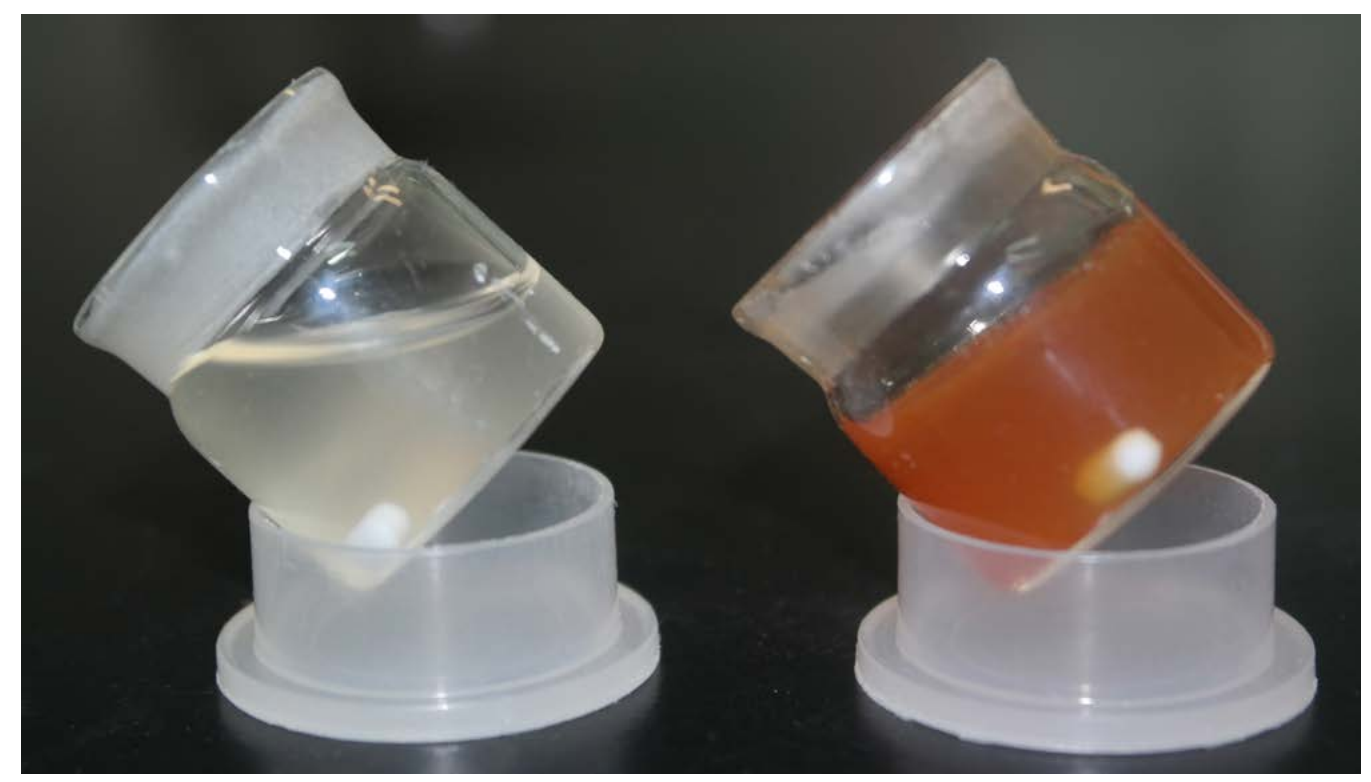

Figure S1. Photograph represents the alginate polymer solution $(2.0 \% \mathrm{w} / \mathrm{w})$ before (left) and after (right) electrochemically controlled oxidation of $\mathrm{Fe}^{2+}$ (35 mM). 


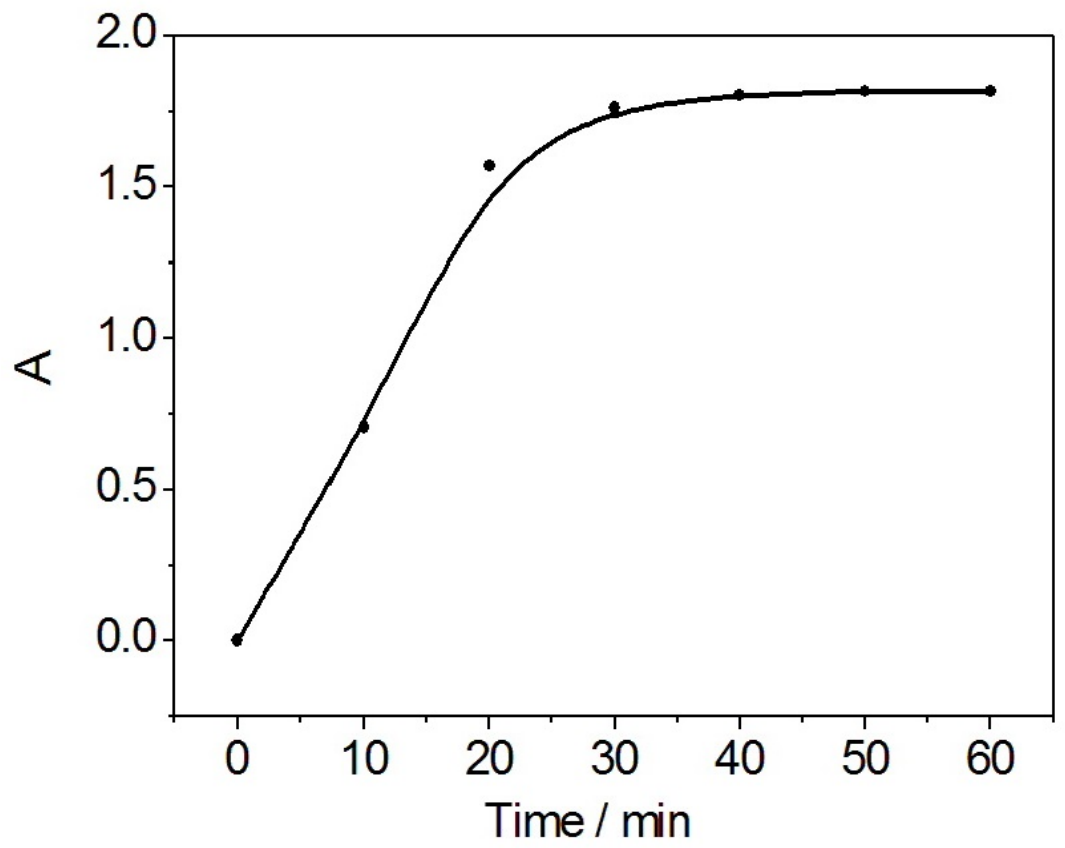

Figure S2. Spontaneous release of HRP from the alginate film with time. The solutions (3.0 mL) of HRP released at different times were collected, to which 4.0 mM ABTS and $2.5 \mathrm{mM} \mathrm{H} \mathrm{H}_{2} \mathrm{O}_{2}$ were then added. After 3 min incubation at room temperature for the ABTS oxidation by $\mathrm{H}_{2} \mathrm{O}_{2}$ in the presence of HRP spontaneously released, the absorbance of the reaction product $\mathrm{ABTS}^{-}$for each solution was measured at $418 \mathrm{~nm}$. 
A
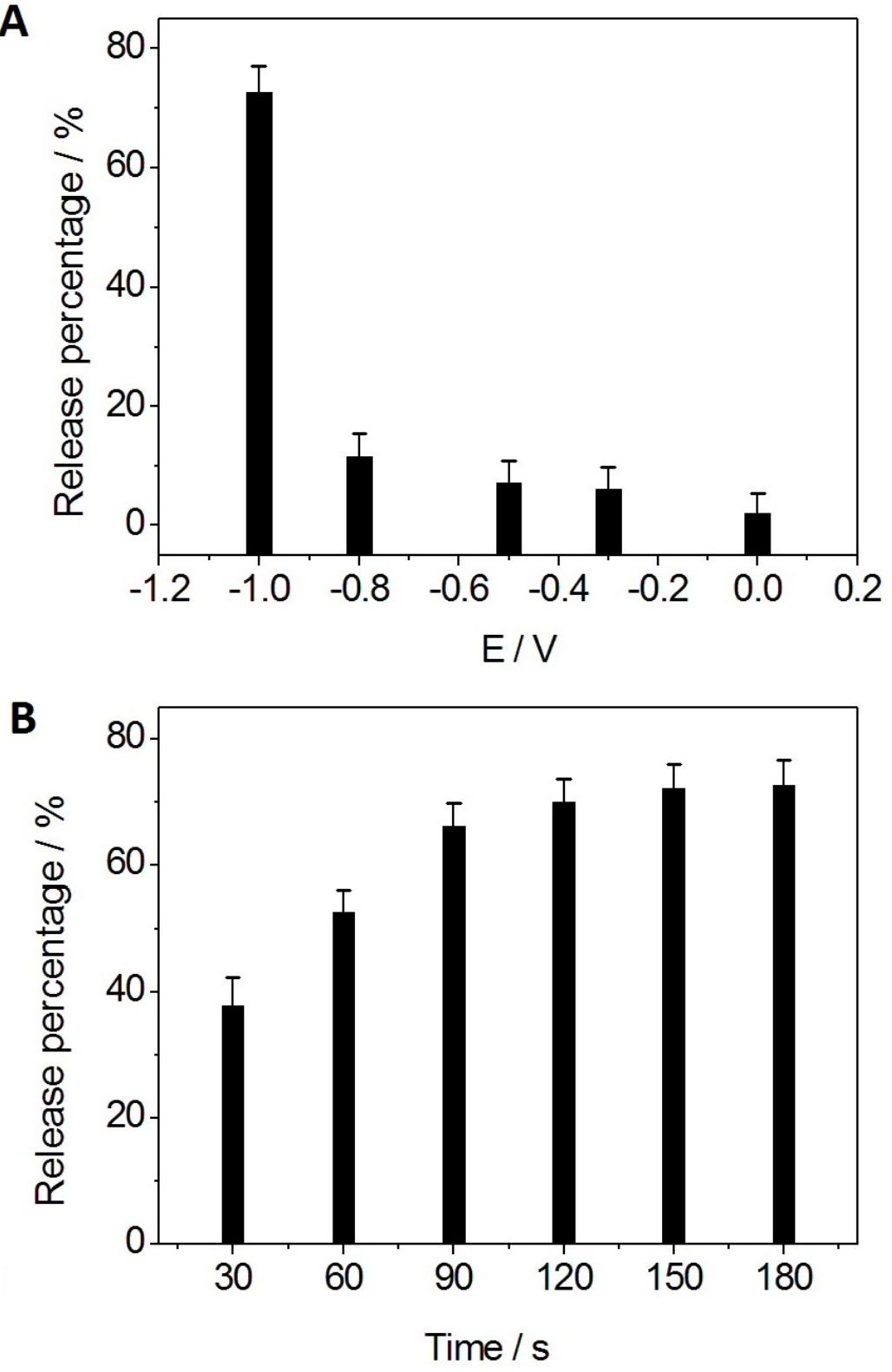

Figure S3. Percentages of the released HRP (from the total amount in the FeAlg film) upon applications of (A) different reductive potentials and (B) different dissolution times. Unless noted otherwise, HRP entrapped in the alginate thin film was electrochemically released at $-1.0 \mathrm{~V}$ for $3 \mathrm{~min}$ in $3.0 \mathrm{~mL} \mathrm{Na}_{2} \mathrm{SO}_{4}$ solution $(0.1 \mathrm{M}) .4 .0$ mM ABTS and $2.5 \mathrm{mM} \mathrm{H}_{2} \mathrm{O}_{2}$ were then added to the solution for the HRP catalyzed oxidation of ABTS. Error bars represent one standard deviation for three measurements. 


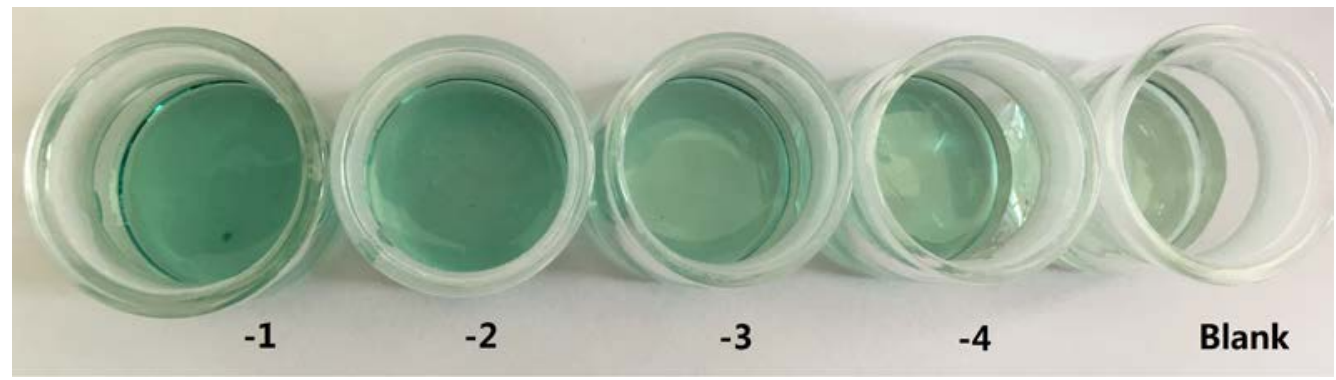

Figure S4. Photograph of the colorimetric responses of the sensing system to $\mathrm{Cl}^{-}$at different concentrations: (-1), $10^{-1} \mathrm{M} ;(-2), 10^{-2} \mathrm{M},(-3), 10^{-3} \mathrm{M} ;(-4), 10^{-4} \mathrm{M}$. HRP entrapped in the alginate thin-films as electrochemically released in $3.0 \mathrm{~mL} \mathrm{Na}_{2} \mathrm{SO}_{4}$ solution (0.1 M, pH 6.0). A reductive potential of $-1.0 \mathrm{~V}$ was applied for $3 \mathrm{~min} .4 .0$ mM ABTS and $2.5 \mathrm{mM} \mathrm{H} \mathrm{H}_{2} \mathrm{O}_{2}$ were then added to the solution for the HRP catalyzed oxidation of ABTS. 